\title{
Quantitative assessment of gait and neurochemical correlation in a classical murine model of Parkinson's disease
}

\author{
Xiao Hong Wang ${ }^{1,2,3 \dagger}$, Gang $\mathrm{Lu}^{2,3 \dagger}$, Xiang Hu${ }^{2,4+}$, Kam Sze Tsang ${ }^{2,5}$, Wing Hang Kwong ${ }^{3}$, Feng Xia Wu ${ }^{1,2}$,
} Hai Wei Meng ${ }^{1}$, Shu Jiang ${ }^{4}$, Shu Wei Liu ${ }^{1^{*}}$, Ho Keung $\mathrm{Ng}^{5}$ and Wai Sang Poon ${ }^{2,3^{*}}$

\begin{abstract}
Background: Gait deficits are important clinical symptoms of Parkinson's disease (PD). However, existing behavioral tests for the detection of motor impairments in rodents with systemic dopamine depletion only measure akinesia and dyskinesia, and data focusing on gait are scarce. We evaluated gait changes in the methyl-4-phenyl-1,2,3,6tetrahydropyridine (MPTP)-induced C57BL/6 murine model of PD by using a computer-assisted CatWalk system. Correlations of gait parameters with tyrosine hydroxylase $(\mathrm{TH})$ protein levels in the substantia nigra $(\mathrm{SN})$ were also investigated.
\end{abstract}

Results: The gait readouts, including the walking duration, variation of walking speed, step cycle, duty cycle, stance, initial dual stance, terminal dual stance, three- and four-point supports, and the base of support between hind limbs was noted to increase significantly one week after MPTP injection. In contrast, values of the stride length, cadence, swing speed, and diagonal dual support decreased substantially following MPTP treatment $(p<0.05)$. All of these changes lasted for three weeks after the last MPTP administration. Except for the stance in the fore limbs and the swing speed in the hind limbs, the gait variability in the PD mice showed a closer correlation with the protein levels of TH in the SN than the walking distances in the conventional open field test. Coordination parameters of the regularity index and step pattern were not affected in mice treated with MPTP.

Conclusion: Data of the study suggest that the computer-assisted CatWalk system can provide reliable and objective criteria to stratify gait changes arising from MPTP-induced bilateral lesions in C57/BL6 mice. The extent of gait changes was noted to correlate with the expression of the biomarker for dopaminergic neurons. This novel analytical method may hold promise in the study of disease progression and new drug screening in a murine PD model.

Keywords: Parkinson's disease, Gait, MPTP, Tyrosine hydroxylase, Neurochemical correlation

\section{Background}

Parkinson's disease (PD) is a pervasive motor disorder resulting from the depletion of dopamine (DA) in the nigrostriatal region of the central nervous system [1]. Gait variability and postural instability are prominent as PD progresses [2]. Rhythmical walking of PD patients is hampered by short stride lengths and low velocities,

\footnotetext{
* Correspondence: Ishw@sdu.edu.cn; wpoon@surgery.cuhk.edu.hk ${ }^{\dagger}$ Equal contributors

'Research Center for Sectional and Imaging Anatomy, Shandong University School of Medicine, 250012 Jinan, Shandong, China

2Division of Neurosurgery, Department of Surgery, Prince of Wales Hospital, The Chinese University of Hong Kong, Hong Kong, China

Full list of author information is available at the end of the article
}

which are related to reduction in the dopamine level $[3,4]$. The postural instability in PD patients often results in falls, when turning around or stepping up, leading to various injuries [5]. The motor deficits are the direct consequence of DA loss in the nigrostriatal system [1]. Tyrosine hydroxylase (TH) is the key enzyme in DA synthesis, as it converts tyrosine into levodopa, and is often used as a biomarker for dopaminergic neurons [6,7].

Methyl-4-phenyl-1,2,3,6-tetrahydropyridine (MPTP) is a neurotoxin being systemically administered to animals for experimental studies of PD [8,9]. It can induce neuropathological changes that mimic those of idiopathic PD in experimental animals, especially in C57/BL6 mice [10,11].

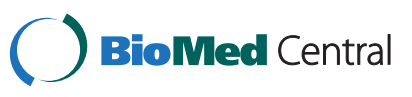


The behavioral tests used in acute, sub-acute, and chronic MPTP-induced animal models include the open field test $[12,13]$, rotarod test $[14,15]$, swimming test $[16]$, and nest building test [17], which results may vary [18-20]. Experimental factors such as timing of testing (day or night), training intention, and testing environment, influence the readouts. Observable changes may be weak and the indicators of these tests, which are usually applied to unilaterally lesioned animals, may not be sufficiently sensitive to demonstrate the functional deficits in bilaterally injured animal models. Besides, animals are tested in an involuntary state and the test indicators, which are limited to either static or dynamic state, cannot adequately display both readouts simultaneously.

Gait analysis has already been described and applied to the assessment of movement disorders in PD [21-24]. Much effort has been made to analyze the gait in animal PD models, nevertheless there is a surprising lack of validation and characterization of gait variability in bilaterally lesioned animal models [23,25-32]. Alexander et al. investigated gait patterns and co-ordinations between limbs of rats subjected to unilateral 6-hydroxydopamine (6-OHDA) lesion by using footprint analysis and ladder walking test [26]. Chuang et al. evaluated gait patterns in a unilaterally 6-OHDA-induced rat model [33]. Fernagut et al. first introduced the manual measurement of stride length using foot prints in bilaterally MPTP-induced lesioned C57/BL6 mice [25]. Goldberg and co-workers assessed stride length, stride frequency, stride duration, variability in stride length, and paw area in MPTP-treated mice having been stressfully trained on treadmill apparatus [23]. Westin et al. investigated gait disorders in 6-OHDAtreated rats by a less commonly used bilateral induction method [34].

In this report we described a novel procedure to validate gait variability in a mouse model of sub-acute $\mathrm{PD}$ of bilateral lesion induced by MPTP. We evaluated the temporal expressions of $\mathrm{TH}$ protein in the $\mathrm{SN}$ and striatum of MPTP-treated mice and assessed the neuro-degeneration in the nigrostriatal regions by studying the correlation of $\mathrm{TH}$ expressions with changes of gait parameters.

\section{Methods}

\section{Animals}

Inbred strain of adult male mice C57BL/6 at aged 8-10 weeks and weighting 25-28 g were used in the study. They were kept in micro-isolator cages with access food and water ad libium in a temperaturecontrolled environment at $21^{\circ} \mathrm{C}$ and 12-hour cycle of light and dark. Animals were amenable daily to the researchers for one week prior to any experiment. Experimental procedures were performed according to the guidelines of the Animal Experiment Ethics Committee of the Chinese University of Hong Kong.

\section{Experiment design}

The design of the study is shown in Figure 1. C57BL/6 mice were allowed to familiarize with the CatWalk device daily and cross the runway in a consistent manner for one week before any experimentation. The mice were then randomly divided into two groups. MPTP-HCl (Sigma, St Louis, MO, USA) at $30 \mathrm{mg}$ per $\mathrm{kg}$ body weight per day was injected intra-peritoneally into 50 mice for five consecutive days to induce PD. Normal saline in equal volume of $\mathrm{MPTP}-\mathrm{HCl}$ was injected into 45 control mice. Upon completion of the administration of either MPTP or normal saline, mice were subjected to computer-assisted CatWalk tests on day 7 and day 21 and open field tests on days 4, 7, 12,16 , and 21 .

Western blotting was performed to analyze TH expressions in the $\mathrm{SN}$ and striatum of mice two days before and on days $2,4,7,12,16$, and 21 after the course of MPTP administration. Immuno-histochemical staining of $\mathrm{TH}$ was conducted to assess the loss of dopaminergic neurons in the $\mathrm{SN}$ and striatum of the mice three weeks post-injection of MPTP.

\section{Gait analysis}

Gait of unforced moving mice was analyzed with the CatWalk XT (Noldus Information Technology, Wageningen, Netherlands). Details have been described previously [35]. CatWalk XT consists of a hardware system of a long, glass walkway plate, illuminated with green light, a high-speed video camera, and a software package for quantitative assessment of animal footprints. Green light is reflected within the glass except at points being touched. It scatters and illuminates the contact area. The intensity of the area of illumination, which is proportional to the exerted pressure, is digitally captured by the video camera and analyzed.

Mice were allowed to walk across the glass walkway in an unforced manner at least six times a day on day 7 prior to injection with either MPTP or normal saline. Mouse tracks that were straight without any interruption or hesitation were treated as successful runs. Runs with any wall climbing, grooming, and staying on the walkway were not analyzed. Mice that failed the CatWalk training were excluded from the study. An average number of 8 replicate crossings (range: 4-6) made by each mouse was recorded. The CatWalk software was used to analyze crossings that had at least five cycles of complete steps. Table 1 shows the definition of the gait and co-ordination parameters used in this study.

\section{Open field test}

Spontaneous locomotor activity in mice was measured using the open field test. Mice were allowed to adapt to the environment for two hours prior to testing. They 


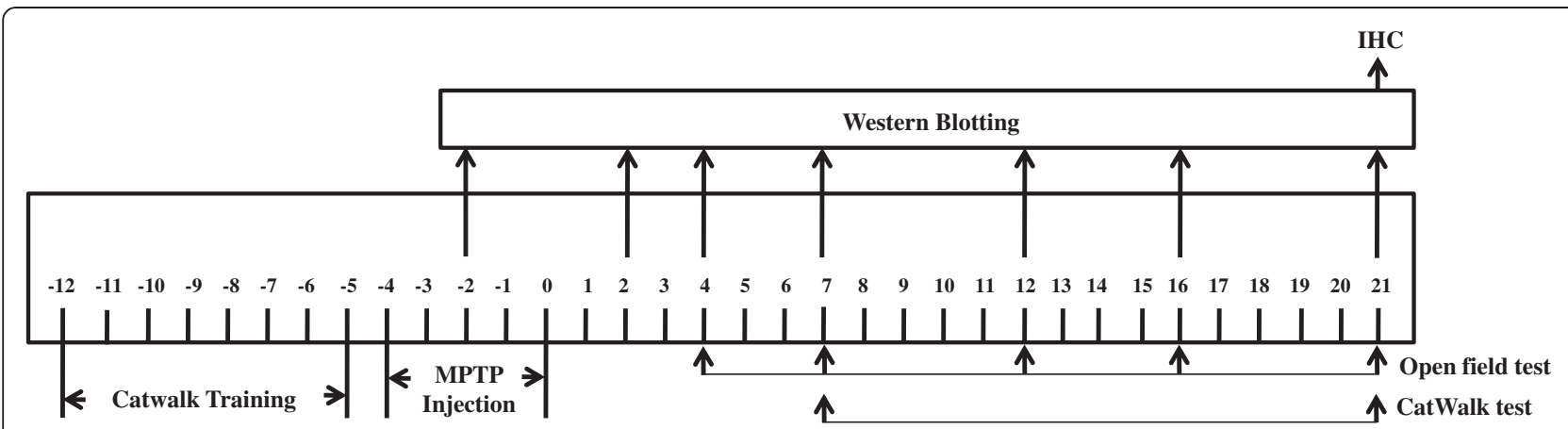

Figure 1 Flow chart of the study.

were then placed individually facing the same wall of a white square box measuring $50 \times 50 \times 25 \mathrm{~cm}$ and kept for 10 minutes under normal lighting. Movements and trajectories of mice were video-taped and analyzed by the Videomex-One software (Columbus Instruments, USA). The box was cleaned with water and $70 \%$ alcohol after each testing to remove the body scent, which could be a cue to move for mice in subsequent evaluations. Eight mice per study arm and time point were recruited.

\section{Tissue processing}

Before and upon completion of MPTP injection on day -2 and days $2,4,7,12,16$, and 21 , respectively, four mice were anesthetized, their thoraxes were cut open, and they were trans-cardiacally perfused with physiologic saline and $4 \%$ paraformaldehyde in $0.1 \mathrm{M}$ of phosphate-buffered saline. Heads were decapitated. Brains were rapidly removed and fixed in $4 \%$ paraformaldehyde for two days at $4{ }^{\circ} \mathrm{C}$, and allowed to further fix in 15\%, 20\%, and 30\% sucrose solution.

\section{Table 1 Definitions of gait parameters}

\begin{tabular}{|c|c|}
\hline Parameter & Definition \\
\hline Run duration & Time of finishing an entire run in second \\
\hline Maximum area & The print area of paw when the braking phase turns into propulsion phase \\
\hline Maximum intensity & Maximum pressure of paw contacting floor \\
\hline Stride length & The distance between two consecutive travels in the same paw \\
\hline Swing duration & The duration in seconds of no contact of a paw with the glass plate \\
\hline Swing speed & The ratio of stride length to swing duration \\
\hline Stance & The time duration in seconds of paws in contact with glass plate \\
\hline Step cycle & The time in seconds between two consecutive initial contacts of the same paw \\
\hline Duty cycle & The percentage of stance over the sum of stance and swing duration \\
\hline Cadence & Steps per second in a trial \\
\hline Base of support & Distance between fore limbs or hind limbs at maximum area \\
\hline Initial dual support & $\begin{array}{l}\text { The first time in a step cycle of a hind paw that the contralateral paw also makes contact } \\
\text { with the glass plate }\end{array}$ \\
\hline Terminal dual support & $\begin{array}{l}\text { The second step in a step cycle of a paw that the contralateral paw also makes contact with } \\
\text { the glass plate }\end{array}$ \\
\hline \multicolumn{2}{|l|}{ Diagonal dual support } \\
\hline \multicolumn{2}{|l|}{ Three-point support } \\
\hline Four-point Support & The relative duration of simultaneous contacts of limbs with the glass plate \\
\hline Step Pattern & Configuration of right $(\mathrm{R})$, left $(\mathrm{L})$, front $(\mathrm{F})$ and hind $(\mathrm{H})$ limbs \\
\hline \multicolumn{2}{|c|}{ Alternate Aa: RF-RH-LF-LH; Ab: LF-RH-RF-LH } \\
\hline \multicolumn{2}{|c|}{ Cruciate Ca: RF-LF-RH-LH; Cb: LF-RF-LH-RH } \\
\hline \multicolumn{2}{|c|}{ Rotary Ra: RF-LF-LH-RH; Rb: LF-RF-RH-LH } \\
\hline Regularity Index & The number of normal step sequence patterns relative to the total number of paw placements \\
\hline
\end{tabular}


The dissecting protocol of Jackson-Lewis and Przedborski was adopted to maximize the isolation of ventral midbrain containing the substantia nigra [36]. The brain was quickly removed and put into a chilled mouse coronal brain matrix (World Precision Instruments Inc., USA). A 2-mm-thick posterior-anterior segment was dissected from the posterior aspect of the cerebral peduncles. The caudal aspect of the dissected section was immediately placed on the disk with the ventral aspect facing the researcher. After the middle brain was separated, the substantia nigra-rich tissue was isolated by cutting at a third of the way from the dorsal midbrain with a blade slanted at a $45^{\circ}$ angle toward the researcher. The striatum was dissected from the brain immediately. All of the brain tissues were stored at $-80^{\circ} \mathrm{C}$ until analysis.

\section{Western blot analysis}

Brain tissues was homogenized in ice-cold lysis buffer containing $50 \mathrm{mM}$ Tris- $\mathrm{HCl}$ at $\mathrm{pH} 6.8,50 \mathrm{mM} \mathrm{NaCl}$, $1 \%$ Triton X-100, $1 \mathrm{mM}$ PMSF, $5 \mu \mathrm{g} / \mathrm{ml}$ of aprotinin, $1 \mu \mathrm{g} / \mathrm{ml}$ of leupeptin and $1 \mu \mathrm{g} / \mathrm{ml}$ of prepstetin (ICN Bio Medical Products, Costa Mesa, CA). The lysates were centrifuged at $12,000 \times g$ for 30 minutes at $4^{\circ} \mathrm{C}$ to separate debris. The protein concentration was assayed using a BIO-RAD DC protein assay kit (BioRad, Hercules, CA). Ten $\mu$ g of protein was used for the western blot analysis.

Protein samples were electrophoresed on 10\% SDSPAGE and blotted onto nitrocellulose membranes (BioRad, Hercules, CA). Having blocked for an hour at room temperature with $20 \mathrm{mM}$ Tris- $\mathrm{HCl}$ at $\mathrm{pH} 7.5,137 \mathrm{mM}$ $\mathrm{NaCl}, 0.05 \%$ Tween 20 and 5\% non-fat milk (BioRad, Hercules, CA). proteins on membranes were incubated with polyclonal rabbit anti-TH (1:3,000, clone AB152, Chemicon International, USA), polyclonal goat anti- $\beta$ actin (1:1,000, Santa Cruz, USA) overnight at $4^{\circ} \mathrm{C}$. Membranes were washed three times and then stained with fluorescein isothiocyanate-conjugated secondary antibody (1:20,000, Rockland, USA) for an hour at room temperature. Upon completion of washings, green fluorescent signals were visualized using the Odyssey ${ }^{\circledR}$ imaging system (LICOR Biosciences, version 3.0). The optical density was read using the Odyssey system.

\section{Immunohistochemical staining of $\mathrm{TH}$}

Cryostat sections of brain slices in cryo-mountant were cut $25 \mu \mathrm{m}$ thick. Floating sections on $0.01 \mathrm{M}$ phosphate buffered saline were exposed to $0.25 \%$ Triton X-100 and $3 \% \mathrm{H}_{2} \mathrm{O}_{2}$, and then blocked in $2 \%$ bovine serum albumin. They were then incubated sequentially with rabbit polyclonal anti-TH antibody (1: 1,000, clone AB152, Chemicon International, USA), biotinylated goat antirabbit antibody (1:200, Vector Laboratories, USA) and horseradish-peroxidase-conjugated avidin-biotin complex (Vector Laboratories, USA). Sections were developed with a DAB kit (Zymed Laboratories Inc, USA). Dark brown signals derived from sections mounted on glass slides were captured and analyzed using a microscope Axioplan 2 (Zeiss, Germany) equipped with a documentation system.

Unbiased stereological counting method was used to enumerate the DA neurons [37]. Every sixth section of the SN was analyzed by means of the optical fractionator and a computer-assisted stereological Olympus Toolbox system version 2.1.4. The $\mathrm{SN}$ of each hemisphere was first delineated using a $4 \mathrm{x}$ objective. A square grid of $150 \times 150 \mu \mathrm{m}$ was randomly super-imposed with and a $100 \times 100 \mu \mathrm{m}$ square dissector counting chamber placed on the first counting area of the image and then systemically moved through all of the counting areas until the entire delineated section was counted. The striatum of each section was located under the same identical condition. Analyses of the $\mathrm{TH}$-immunoreactive profiles were restricted to the $\mathrm{SNpc}$ and thus excluded the ventral tegmental area. The optical density of the THimmuno-reactive fibers was evaluated by the software Metamorph 6.3 on 9-11 sections spanning the striatum. The background signal captured from the corpus callosum was subtracted from the readout of the optical density of each striatal section. Scorings of TH-immuno-reactivity and optical density were performed by two researchers in a blind manner. Results derived from 9-11 sections were averaged.

\section{Statistical analysis}

All the data were expressed as mean \pm standard error of mean (SEM). One-way ANOVA was used for the statistical evaluation of gait changes in the CatWalk test. Repeatedmeasure one-way ANOVA was used to statistically analyze the path lengths in the open field test at various time intervals after MPTP injection. The correlations of the $\mathrm{TH}$ protein levels in the $\mathrm{SN}$ with the motor parameters in the CatWalk test and open field test were evaluated by the Pearson's product-moment correlation coefficient. Differences in the expression levels of $\mathrm{TH}$ protein at different time points were analyzed by ANOVA and the StudentNewman-Keuls post hoc test. Quantitative differences of $\mathrm{TH}$-immuno-reactive cells in $\mathrm{SN}$ were evaluated using the Student's t-test. A $p$ value $\leq 0.05$ was considered as statistically significant. All of the data were analyzed using the SPSS 17.0 software (SPSS Inc., Chicago, USA).

\section{Results}

\section{Catwalk test}

Gait of mice was evaluated with CatWalk system at one week and three weeks upon completion of the administration of either MPTP or normal saline. A decrease in the stride length and an increase in variation of stance and swing were noted in the MPTP-treated mouse compared to the control mouse. Besides, A fall in diagonal 
dual support and an upsurge of four- and three-point supports were evident (Figure 2). Abnormal gait persisted over the three-week study period. A mean \pm SEM of walking duration taken by eight mice on day 21 postMPTP treatment was significantly longer than that of eight control mice having normal saline injection (time in seconds: MPTP-induced PD mice vs. control mice; $3.099 \pm$ 0.689 seconds vs. $1.708 \pm 0.308$ seconds; $p \leq 0.01$, Figure 3A). The extent of variation in walking speed of MPTP-treated mice was more profound than that of the control mice (MPTP-induced PD mice vs. control mice; $34.92 \pm 3.61 \%$ vs. $24.44 \pm 2.49 \%, p=0.019$, Figure $3 \mathrm{~B})$. The cadence of MPTP-treated mice dropped significantly (steps per second: MPTP-induced PD mice vs. control mice; $15.18 \pm 0.52$ vs. $19.19 \pm 0.68 ; p \leq 0.05$, Figure $3 C$ ).

The swing speeds of paws deteriorated markedly (Figure 3D) and the stride lengths of limbs decreased significantly (Figure 3E), whereas the stances, duty cycles, and step cycles of paws increased substantially (Figures 3 $\mathrm{F}-\mathrm{H})$. The initial dual stance and terminal dual stance of paw were also prolonged in MPTP-treated mice compared with those of control counterparts (Figure 4A-B). A relative decrease in diagonal dual support was noted in MPTP-treated mice (incidence of diagonal dual support: MPTP-induced PD mice vs. control mice; $59.76 \pm 3.06 \%$ vs. $80.52 \pm 1.77 \%, p \leq 0.0001$, Figure 4 C). Significant increases of three-point support and four-point support were noted in MPTP-treated mice (incidence of threepoint support: PD mice vs. control mice; $25.99 \pm 3.26 \%$ vs. $8.07 \pm 1.62 \% ; p \leq 0.0001$; Figure $4 \mathrm{D}$, incidence of four-point support: PD mice vs. control mice; $7.33 \pm 1.3 \%$ vs. $1.24 \pm 0.47 \% ; p \leq 0.0001$; Figure $4 \mathrm{E})$. The bases of support between hind limbs were relatively longer (PD mice vs. control mice; $4.12 \pm 0.08 \mathrm{~cm}$ vs. $3.63 \pm$ $0.06 \mathrm{~cm} ; p \leq 0.0001$; Figure 4F).

On the other hand, readouts of gait parameters of maximum pressure intensity, maximum contact area, and swing duration of paws of MPTP-treated mice and control mice are comparable (data are not shown). There was also no significant difference in inter-limb coordination among PD mice and normal mice.

Step patterns and regularity index are indices of coordination, and in these two measures there were no differences between the treated group and the controls. It may be that the motor dysfunction induced by the nigrostriatal lesion in this study did not affect the coordination of the tetrapods. This supposition is supported by the results of Chuang and his colleague [33].

\section{Open field test}

The locomotor activity was measured by means of the open field test to investigate the time course of changes in spontaneous activity induced by the MPTP. Repeatedmeasure one way ANOVA revealed the effect of the number of times of repetitive tests $(\mathrm{F} 4,11=106.6$; $\mathrm{p}<0.001)$ and the treatment interaction $(\mathrm{F} 4,11=42.865$; $\mathrm{P}<0.001)$, indicating that MPTP treatment affected spontaneous activity compared with saline treated mice (Figure 5). The distances travelled by mice on day 4 and at three weeks after the last injection of MPTP were significantly less than those of the vehicle-treated mice $(p<0.001)$.
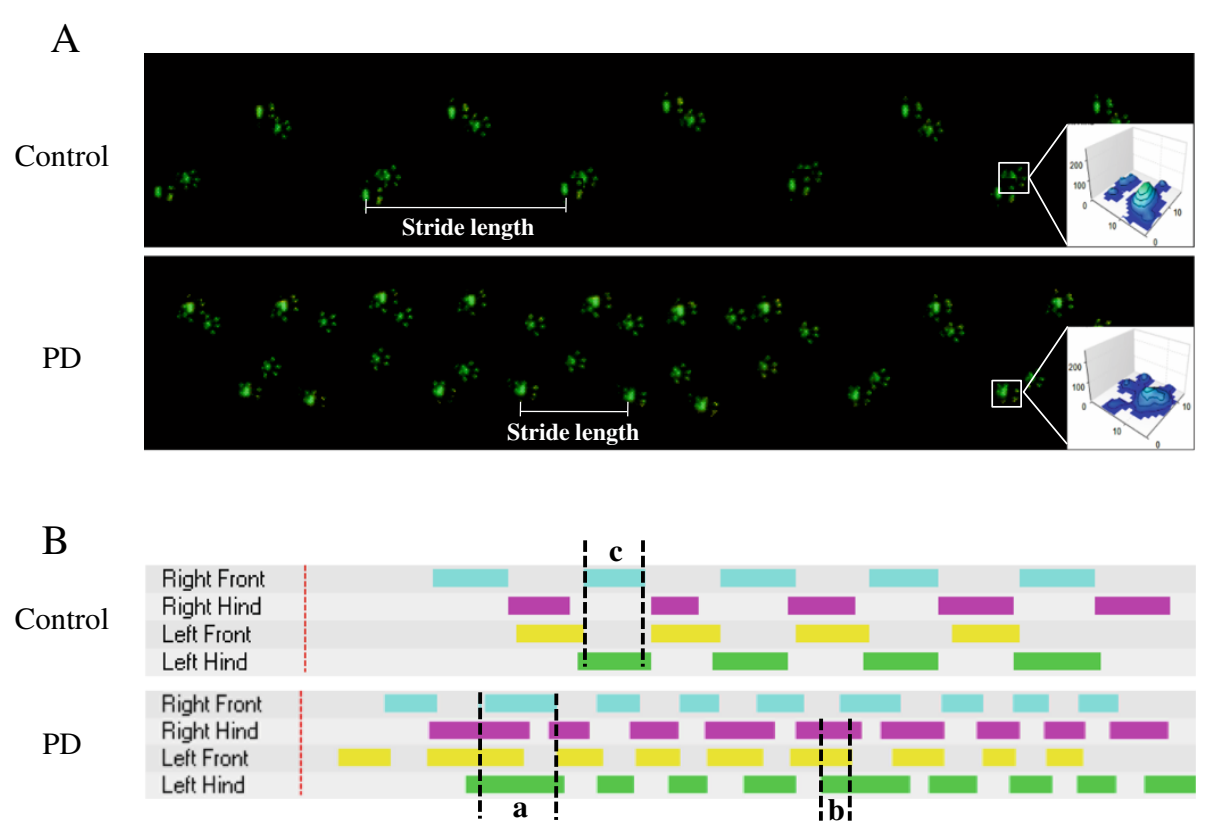

Figure 2 Representative illuminations of footprints. 


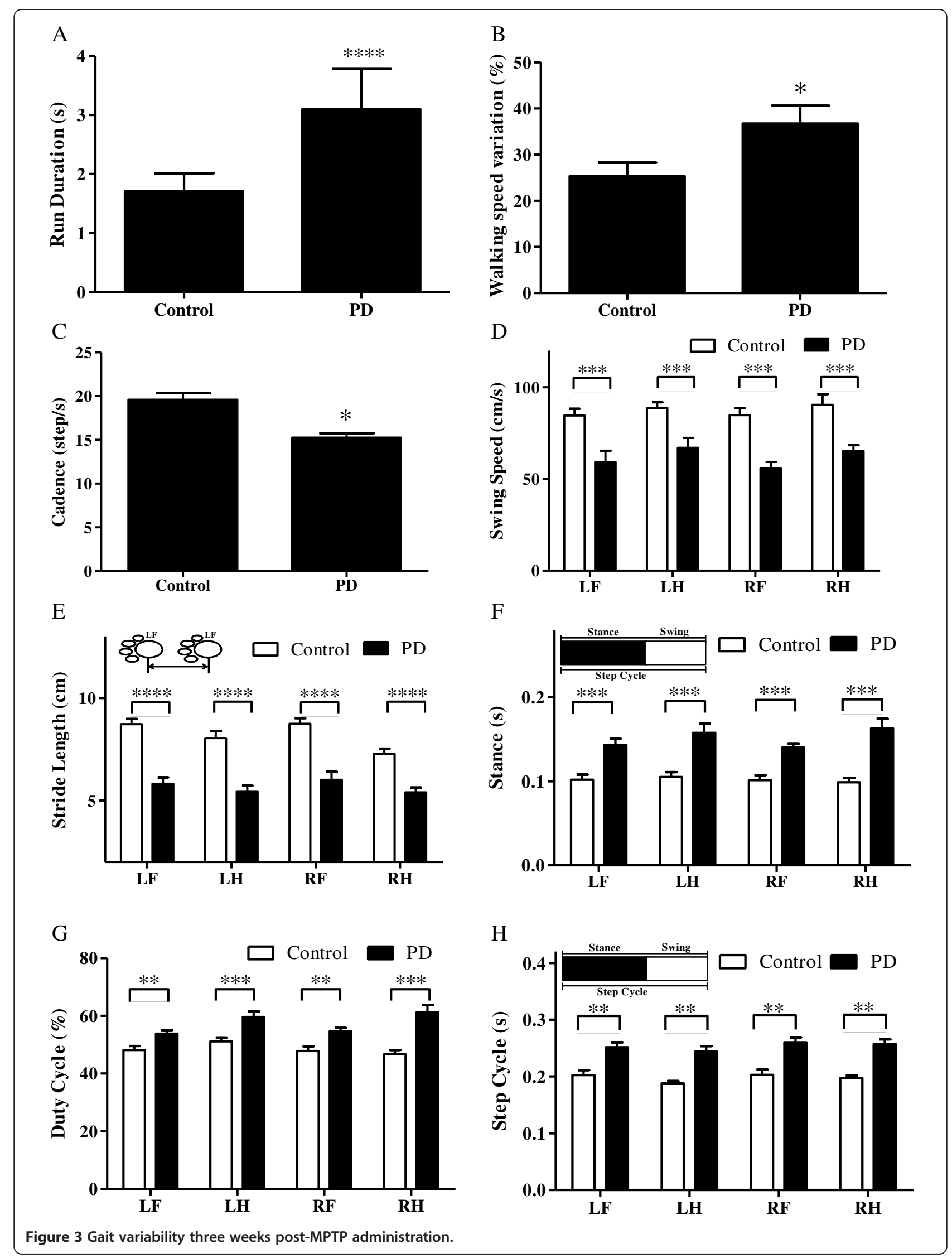




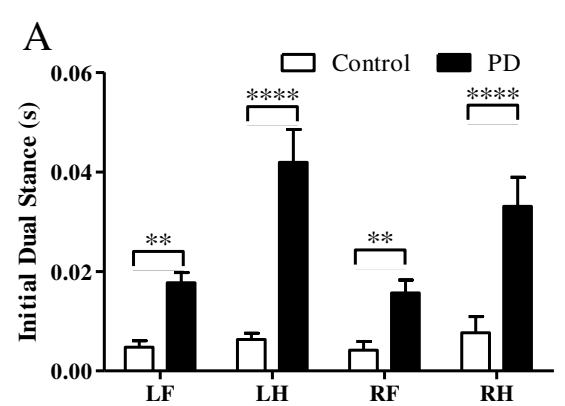

C
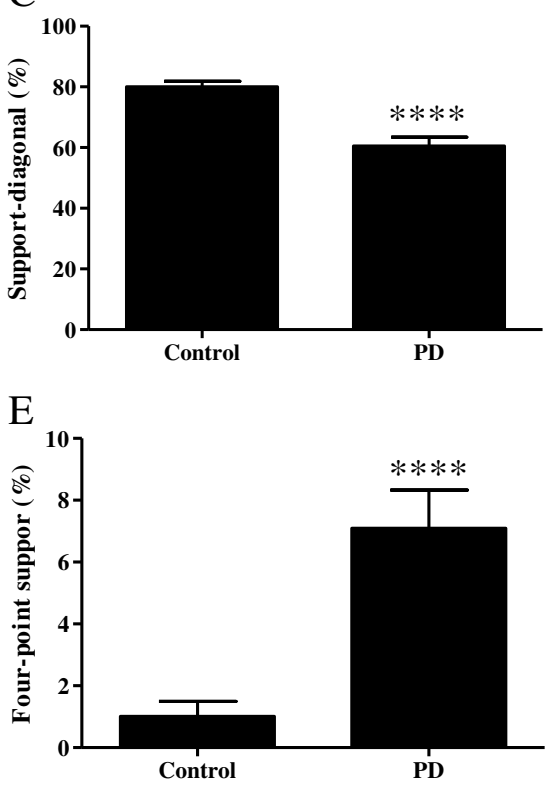

Figure 4 Gait changes three weeks post-MPTP administration.

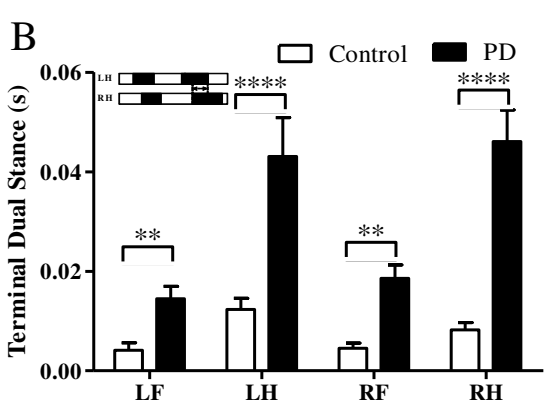

$\mathrm{D}$

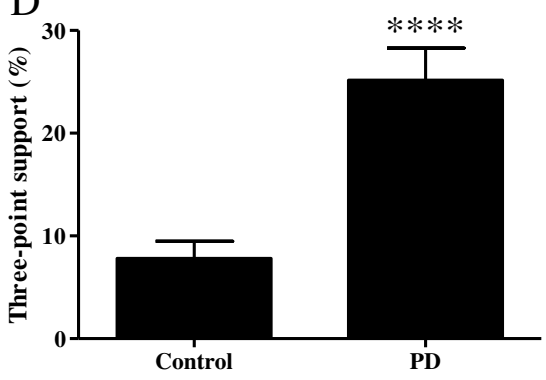

$\mathrm{F}$

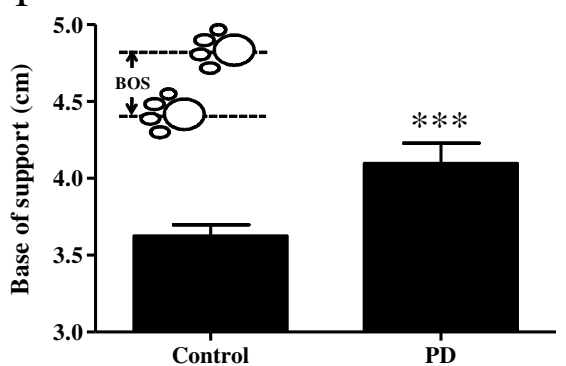

Expression and correlation of $\mathrm{TH}$ with gait variability

A significant fall in $\mathrm{TH}$ was evident in both substantia nigra and striatum of mice starting on day 2 of MPTP administration, followed by a slight rebound at two weeks. TH then remained at the lowest levels on three weeks post-MPTP injection. It was noted that the downregulation of $\mathrm{TH}$ by $\mathrm{MPTP}$ in the striatum was more profound than that in the substantia nigra (Figure 6).

Table 2 shows the significantly positive correlations of the levels of TH in the substantia nigra of MPTP-treated mice with readouts derived from CatWalk tests of diagonal dual support, stride length in all limbs, and swing speed in the forelimbs three weeks post MPTP administration. Substantially negative correlations of TH levels with stance in the hind limbs, step cycle, duty cycle, initial dual stance, terminal dual stance, three-point support, four-point support, walking speed variation, cadence, and base of support between hind limbs were noted. On the other hand, the levels of TH correlated poorly with the trajectories in the open field tests.

\section{Histological markers of nigrostriatal degeneration} induced by MPTP

Immuno-histochemical staining of $\mathrm{TH}$ demonstrated a substantial loss of immuno-reactivity in the SN and striatum of mice three weeks post-MPTP administration (Figure 7). Unbiased stereological counting displayed a significant loss of up to $57.04 \%$ compared with the control group on the $21^{\text {st }}$ day after the last injection of MPTP $(p<0.05)$. Injection with MPTP also resulted in a great depletion of $\mathrm{TH}$-ir in the striatum of up to $61.32 \%$ of that in control group on the $21^{\text {st }}$ day post-treatment. Data suggest that deprivation and denervation of TH-immunoreactive neurons and terminals might be attributed to the gait variability experienced by MPTP-treated mice.

\section{Discussion}

Quantitative assessment of gait

In this study, we developed a time-efficient and animalfriendly CatWalk test of motor functions in an MPTPinduced PD mouse model. Both static and dynamic 

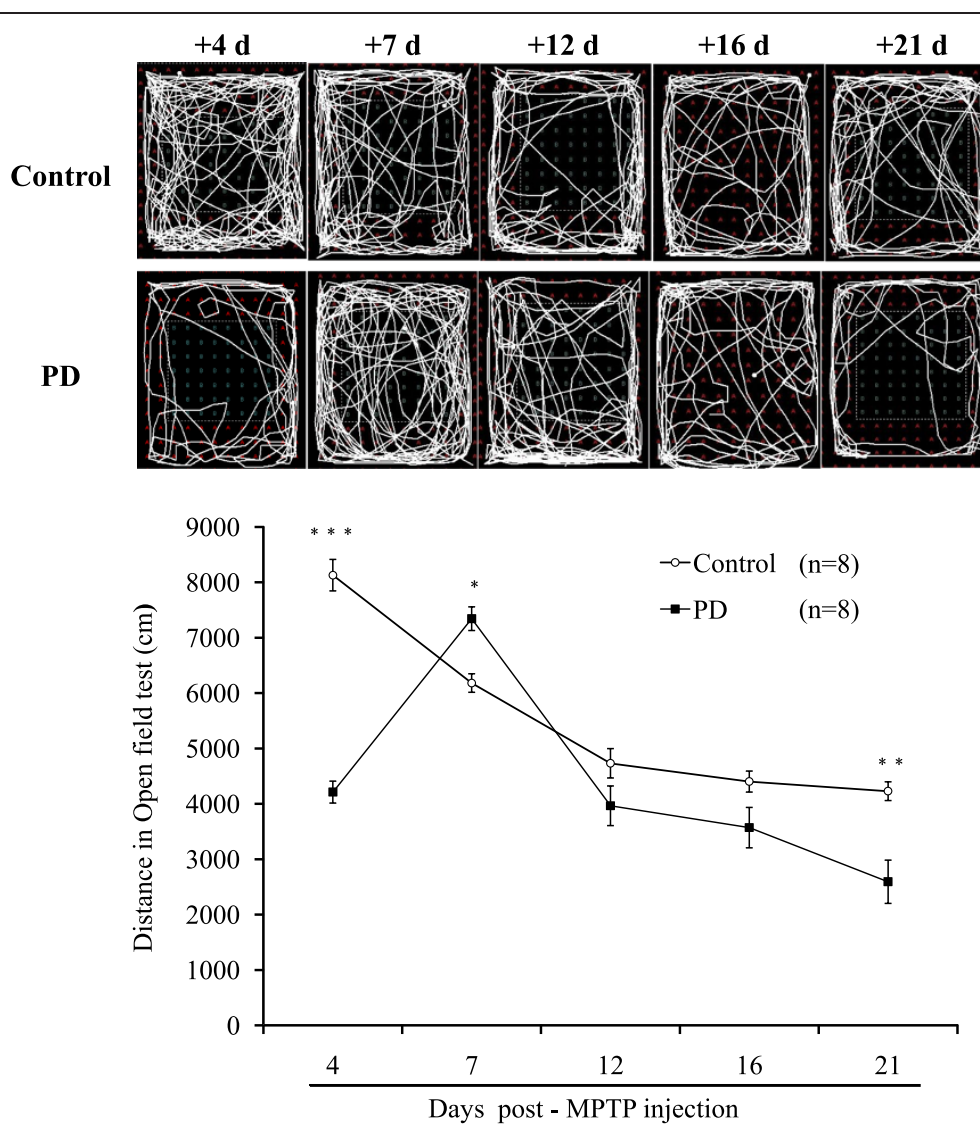

Figure 5 Representative trajectories and locomotor activity in open field test.

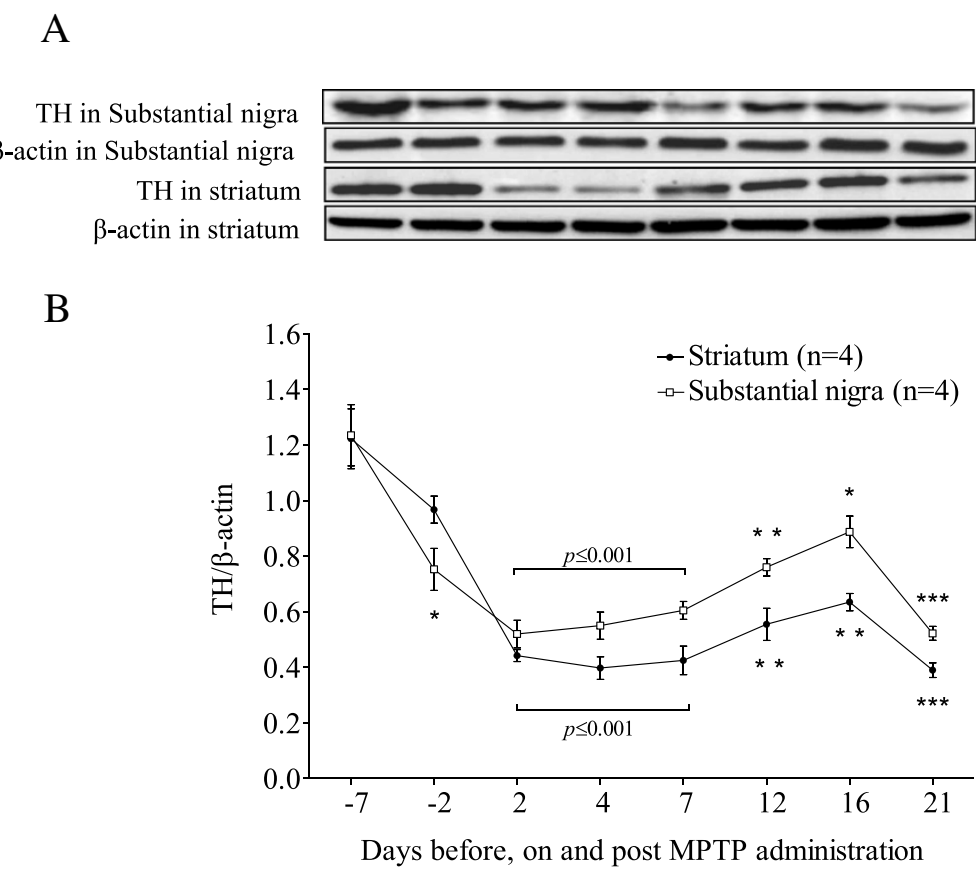

Figure 6 Western immunoblotting of tyrosine hydroxylase (TH) in the substantia nigra and striatum of mice before, on and post MPTP administration. 
Table 2 Correlation of motor deficits with tyrosine hydroxylase expression in substantia nigra three weeks post MPTP administration

\begin{tabular}{|c|c|c|c|c|c|}
\hline Parameters & $\begin{array}{l}\text { Pearson correlation } \\
\text { coefficient }\end{array}$ & $P$-value & Parameters & $\begin{array}{l}\text { Pearson correlation } \\
\text { coefficient }\end{array}$ & $P$-value \\
\hline Stance & & & Duty cycle & & \\
\hline LF & -0.183 & 0.235 & LF & -0.444 & 0.003 \\
\hline $\mathrm{LH}$ & -0.420 & 0.003 & $\mathrm{LH}$ & -0.594 & 0.0001 \\
\hline RF & -0.191 & 0.215 & RF & -0.422 & 0.004 \\
\hline $\mathrm{RH}$ & -0.447 & 0.002 & $\mathrm{RH}$ & -0.717 & 0.0001 \\
\hline Stride length & & & Initial dual support & & \\
\hline LF & 0.759 & 0.0001 & LF & -0.385 & 0.01 \\
\hline $\mathrm{LH}$ & 0.725 & 0.0001 & $\mathrm{LH}$ & -0.466 & 0.001 \\
\hline RF & 0.660 & 0.0001 & RF & -0.354 & 0.019 \\
\hline $\mathrm{RH}$ & 0.568 & 0.0001 & $\mathrm{RH}$ & -0.419 & 0.001 \\
\hline Swing speed & & & Terminal dual support & & \\
\hline LF & 0.393 & 0.008 & LF & -0.455 & 0.002 \\
\hline $\mathrm{LH}$ & 0.252 & 0.099 & $\mathrm{LH}$ & -0.385 & 0.010 \\
\hline RF & 0.359 & 0.007 & RF & -0.497 & 0.001 \\
\hline $\mathrm{RH}$ & 0.288 & 0.058 & $\mathrm{RH}$ & -0.357 & 0.022 \\
\hline Step cycle & & & Diagonal dual support & 0.677 & 0.0001 \\
\hline LF & -0.463 & 0.001 & Three-point support & -0.589 & 0.0001 \\
\hline $\mathrm{LH}$ & -0.301 & 0.046 & Four-point support & -0.455 & 0.002 \\
\hline RF & -0.453 & 0.002 & Speed variation & -0.469 & 0.001 \\
\hline $\mathrm{RH}$ & -0.368 & 0.014 & Cadence & -0.346 & 0.021 \\
\hline Distance in open field test & 0.206 & 0.358 & Base of support between hind limbs & -0.569 & 0.001 \\
\hline
\end{tabular}

LF: left front, LH: left hind, RF: right front, RH: right hind.
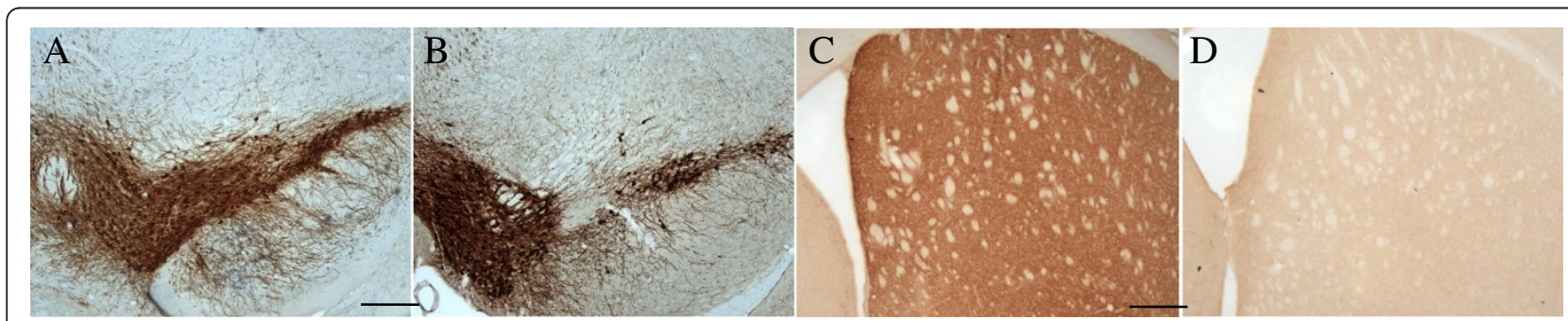

$\mathrm{E}$

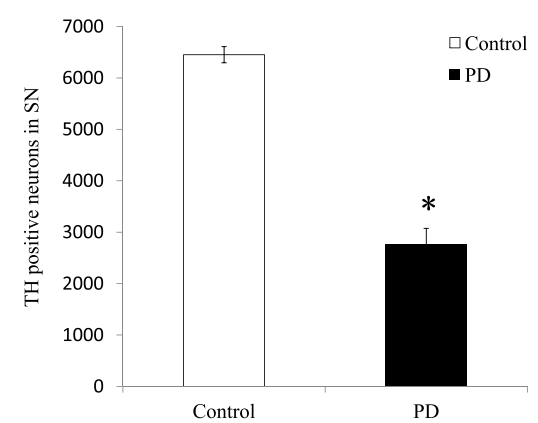

F

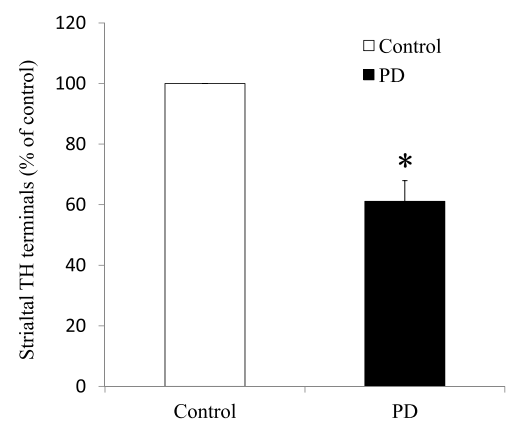

Figure 7 Enumeration of neurons expressing tyrosine hydroxylase (TH) in the substantia nigra and striatum of mice three weeks post-MPTP administration. 
parameters of gait variability were collected simultaneously. Static parameters of stance, print length, print width, print area, maximum contact intensity, and maximum contact area are based upon contacts of individual paw with the glass plate of walkway. Dynamic parameters are walk duration, variation of walk speed, swing speed, and base of support between limbs. The study showed that the CatWalk test was able to sensitively assess gait disorder and inter-limb coordination deficit in a mouse model of PD with bilateral lesion of DA neurons.

There are many behavioral tests in MPTP-induced PD mouse model. These have to be robust and sensitive, in order to detect functional changes, days and even weeks after challenge by a neuro-toxin. The widely employed rotarod test is a feasible means to analyze coordination disorders in a bilaterally lesioned PD mouse model, but it is insensitive. It was noted that the progress of preanalytical training of animals may recruit DA in the striatum [38]. The pole-, grid- and nest-building tests are sensitive to striatal DA level in MPTP-treated mice, but are heavily dependent on the skilled manipulation of fore-limbs of the animal being studied $[17,39,40]$.

The open field test is the most commonly used method to evaluate motor deficits because of its ease and rapidity of use and rapidity in animals following MPTP insult [12,41-45]. We conducted open field test to study motor deficits in mice three weeks after induction of sub-acute neural injury and applied the open field test as the control in the evaluation of the CatWalk test. Readouts of the open field test in the study agree with previous reports of significant motor deficits in mice a few days after MPTP injection $[43,44]$. A significant upsurge of trajectories was also noted in mice one week post-MPTP administration compared to those read on day 4 and that of control mice on day 7 . The scenario might be attributable to an increase of DA metabolism and fluctuation of catecholamines such as 5-hydroxytryptamine in the midbrains of mice induced by dopaminergic neurotic and MPTP and a booster of muscle strength to balance posture instability [46-49].

Although open field test is a friendly test, the time points to detect locomotor changes in animals are limited. Here, we could only apply the time points of the 4 day and three weeks after MPTP injury for the functional evaluation in the open field test. The stability of behavioral performance of animals in the open field is vulnerable to the toxic effects of MPTP on biogenic amines inside and outside the brain [44], administration regimens and dosages of MPTP $[48,50]$ and environments.

The computer-assisted CatWalk test paralleled the motor function assessment in the open field test by collecting static and dynamic parameters in non-stressed rodents to investigate gait variability and coordination changes simultaneously. The stride length, run duration, stance, step cycle, duty cycle, swing speed, and cadence can be used to assess gait changes. The base of support between limbs, initial dual stance, terminal dual stance and diagonal dual support, three-point support and four-point support reflects the posture alterations. The regularity index and step sequence evaluates inter-limb coordination. Animals therefore do not need to undergo any complex process of training. They are allowed to simply run freely along the glass walkway four to six times. Data from the study show that their abnormal gait , in terms of stride length, duty cycle, dual diagonal support, three-point support, four-point support, and base of support between limbs persisted up to three weeks post-MPTP administration. The parameters of inter-limb coordination, regularity index, and step pattern showed no significant difference in MPTP-treated mice, compared to those of control mice. The extent of MPTP-mediated nigrostriatal lesion might not elicit any observable discoordination of tetrapod [33]. The observation may also be related to stronger postural adjustment in quadrupeds than in bipeds.

\section{TH profiling at time of MPTP insult}

MPTP-mediated dopaminergic cell loss occurred predominantly in the $\mathrm{SN}$, whereas DA neurons in the ventral tegmental area were less affected $[8,51]$.

The remarkable reproducible neuroplasticity of the nigrostriatal dopaminergic neurons after MPTP injury has been reported in mice [52]. The decrease in the expression of $\mathrm{TH}$ protein was noted to correlate with the loss of DA neurons in the SN due to acute MPTP injury [9]. It is likely that the decline in striatal dopamine content was due to the decrease in the expression of $\mathrm{TH}$ protein as a consequence of MPTP lesion. The rescue of $\mathrm{TH}$ protein is one of the determinants for regaining striatal dopamine in surviving nigrostriatal dopaminergic neurons [40-42]. Readouts of western blotting in the study demonstrated that protein levels of $\mathrm{TH}$ in the $\mathrm{SN}$ and striatum appeared to rebound two weeks post-MPTP administration; however the expressions were still significantly lower than those derived from intact $\mathrm{SN}$ and striatum.

The study data were in line with those reported previously by Ookubo et al. [53]. The TH protein level fell to the lowest extent after three weeks. The stable lesion in PD mice can serve as a proxy for Parkinsonism in mice, but not for the assessment of MPTP neurotoxicity. The upregulated expression of TH protein expression two weeks after MPTP injury might have a robust compensatory increase resulted by surviving nigrostriatal dopaminergic neurons against the neurotoxicity of the MPTP $[54,55]$. The study data suggest that the open field test is insensitive to the extent of DA lesion except on day 4 and three weeks post-MPTP challenge. 


\section{Translational investigation of gait variability in MPTP- treated mice}

The study provided a thorough analysis of the very fine locomotor aspects in a mouse model of sub-acute PD mediated by MPTP. The CatWalk test revealed detailed gait changes in mice at one week and three weeks postMPTP challenge. That stride length decreased markedly in MPTP-treated mice may be the result of an increase in muscle rigidity and hypokinesia [40,56,57]. Results of the study were in accordance with previous studies $[23,27,34,58]$ and reports on PD patients [59-61], suggesting that stride length serves as a successful translational parameter for PD.

Bradykinesia is one of the major hallmarks of PD $[59,62]$. Run duration, speed variation, swing speed, and step cycle are parameters related to velocity in mobility analysis. Using the CatWalk analysis system, we identified a significant increase in walking duration accompanied with a great variation in the walking speed in MPTPtreated mice. The greater variation in walking speed, which is similar to the fractal-like gait in PD, suggests possible marked inconsistencies in the timing of gait and inadequate postural adjustments [63-65]. The step cycle was increased in the MPTP-treated mice of the study due mainly to the longer contact between the paws and the glass plate. Results of the study were in line with a previous gait assessment in a bilateral 6-OHDA rat model [34]. Gait cadence decreased significantly in MPTP-treated mice. However, an increase in step frequency was noted in PD [66,67]. Cadence varies directly with stride length and inversely with the step cycle. The decrease in stride length noted in MPTP-treated mice of the study might not counter-balance the increase in the step cycle. As a consequence, the cadence decreased in MPTP-treated mice.

Support, either temporal or spatial, is another aspect related to gait in PD. The significant increases of initial dual stance, terminal dual stance, three-point support, and four-point support and the substantial decrease in diagonal dual support in the study suggested an increased duration of the postural phase in the MPTPtreated mice. The study data agree with reports on a longer double limb support time in PD, which may stem from the long time taken to prepare for the generation of propulsive forces $[3,68]$. Results are in line with delays of freezing of gait or gait hesitation in PD [69-71]. The increased duration of the postural phase and the decrease in propulsive forces during the postural and movement phases resulted in a shorter step length and a slower step velocity. The spatial parameters of base of support between hind limbs increased substantially in MPTP-treated mice but there was no obvious difference in base of support between fore limbs. This might be related to hind limb rather than fore limb compensation, at least in part, playing a role in the gait instability of
MPTP-treated mice in the study. On the other hand, the study data contrast with the findings of Westin et al. in which the base of support between fore limbs decreased significantly after 6-OHDA injury. This might be attributed to the difference in injured cerebral areas of animals in the two studies [34].

Interlimb coordination parameters of regularity index and step pattern were also studied. There are three categories in the step patterns: cruciate $(\mathrm{Ca}, \mathrm{Cb})$, alternate $(\mathrm{Aa}, \mathrm{Ab})$, and rotary $(\mathrm{Ra}, \mathrm{Rb})$. The 'Ab' alternate pattern is the most common step cycle, constituting $80 \%$ to $95 \%$ of the total step cycles in intact rodents, the other patterns are very rare in intact animals. The Ab pattern remains dominant in the Parkinson's disease animal model [72]. In this study, there was a slight but not significant decrease in the incidence of this step pattern in mice after MPTP injury. This result is supported by Chuang [33], and may be related to stronger postural adjustment in quadrupeds than in bipeds.

\section{Correlation between the gait parameters and TH levels in the SN}

Various strategies have been employed to verify the correlation between the motor deficits and the impairment of the nigrostriatal system. Here, we used correlation analysis of the motor parameters and $\mathrm{TH}$ protein levels in the SN. Compared with the travel distance measured in the open field test, parameters of the run duration, stride length, duty cycle, swing speed, base of support between hind limbs, three-point support, and four-point support in the CatWalk test were much more strongly correlated with the SN TH protein level. This is one of the first studies to apply correlation analysis to gait measures and $\mathrm{TH}$ protein levels in the SN to further confirm gait and posture deficits in the classic sub-acute MPTP PD model.

\section{Conclusion}

This study reports novel and low-stress method of analyzing the motor functions of mice, which may be applied to monitor gait variability related to disease progression and treatment strategy.

\section{Competing interests}

The authors declare that they have no conflicts of interests.

\section{Authors' contributions}

X Hu, G Lu, and XH Wang carried out the CatWalk and open field tests, collected the data, and drafted the manuscript. WS Poon, SW Liu, and $\mathrm{KH}$ Kwong contributed to the study design, discussed the results, and revised the manuscript. FX Wu, HW Meng, and KS Tsang performed the Western Blotting and histological analyses. FX Wu, S Jiang, and HK Ng carried out the model establishment and evaluation. All authors read and approved the final manuscript.

\section{Author details}

${ }^{1}$ Research Center for Sectional and Imaging Anatomy, Shandong University School of Medicine, 250012 Jinan, Shandong, China. ${ }^{2}$ Division of 
Neurosurgery, Department of Surgery, Prince of Wales Hospital, The Chinese University of Hong Kong, Hong Kong, China. ${ }^{3}$ School of Biomedical Sciences, The Chinese University of Hong Kong, Hong Kong, China. ${ }^{4}$ Shenzhen Beike Cell Engineering Research Institute, Shenzhen, China. ${ }^{5}$ Department of Anatomical and Cellular Pathology, The Chinese University of Hong Kong, Hong Kong, China.

Received: 28 November 2011 Accepted: 14 September 2012 Published: 14 November 2012

\section{References}

1. Fearnley JM, Lees AJ: Ageing and Parkinson's disease: substantia nigra regional selectivity. Brain 1991, 114(Pt 5):2283-2301.

2. Lewis GN, Byblow WD, Walt SE: Stride length regulation in Parkinson's disease: the use of extrinsic, visual cues. Brain 2000, 123:2077-2090.

3. Morris ME, lansek R, Matyas TA, Summers JJ: Stride length regulation in Parkinson's disease. Normalization strategies and underlying mechanisms. Brain 1996, 119(Pt 2):551-568.

4. Morris ME, lansek R, Matyas TA, Summers JJ: Ability to modulate walking cadence remains intact in Parkinson's disease. J Neurol Neurosurg Psychiatry 1994, 57:1532-1534.

5. Visser JE, Nijhuis LBO, Janssen L, et al: Dynamic Posturography in Parkinson's Disease: Diagnostic Utility of the "First Trial Effect". Neuroscience 2010, 168:387-394.

6. Dunkley PR, Bobrovskaya L, Graham ME, von Nagy-Felsobuki El, Dickson PW: Tyrosine hydroxylase phosphorylation: regulation and consequences. J Neurochem 2004, 91:1025-1043

7. Goldstein M, Lieberman A: The role of the regulatory enzymes of catecholamine synthesis in Parkinson's disease. Neurology 1992, 42:8-12. discussion 41-18.

8. Burns RS, Chiueh CC, Markey SP, et al: A primate model of parkinsonism: selective destruction of dopaminergic neurons in the pars compacta of the substantia nigra by N-methyl-4-phenyl-1,2,3,6-tetrahydropyridine. Proc Natl Acad Sci USA 1983, 80:4546-4550.

9. Jackson-Lewis $\mathrm{V}$, Jakowec M, Burke RE, Przedborski S: Time course and morphology of dopaminergic neuronal death caused by the neurotoxin 1-methyl-4-phenyl-1,2,3,6-tetrahydropyridine. Neurodegeneration 1995, 4:257-269.

10. Heikkila RE, Manzino L, Cabbat FS, Duvoisin RC: Protection against the dopaminergic neurotoxicity of 1-methyl-4-phenyl-1,2,5,6tetrahydropyridine by monoamine oxidase inhibitors. Nature 1984 311:467-469.

11. Heikkila RE, Hess A, Duvoisin RC: Dopaminergic neurotoxicity of 1-methyl4-phenyl-1,2,5,6-tetrahydropyridine in mice. Science 1984, 224:1451-1453.

12. Biju K, Zhou Q, Li G, et al: Macrophage-mediated GDNF delivery protects against dopaminergic neurodegeneration: a therapeutic strategy for Parkinson's disease. Mol Ther 2010, 18:1536-1544.

13. Pothakos K, Kurz MJ, Lau YS: Restorative effect of endurance exercise on behavioral deficits in the chronic mouse model of Parkinson's disease with severe neurodegeneration. BMC Neurosci 2009, 10:6.

14. Vijitruth R, Liu M, Choi DY, et al: Cyclooxygenase-2 mediates microglial activation and secondary dopaminergic cell death in the mouse MPTP model of Parkinson's disease. J Neuroinflammation 2006, 3:6

15. Gorton LM, Vuckovic MG, Vertelkina N, et al: Exercise effects on motor and affective behavior and catecholamine neurochemistry in the MPTP-lesioned mouse. Behav Brain Res 2010, 213:253-262.

16. Ferro MM, Bellissimo Ml, Anselmo-Franci JA, et al: Comparison of bilaterally 6-OHDA- and MPTP-lesioned rats as models of the early phase of Parkinson's disease: Histological, neurochemical, motor and memory alterations. J Neurosci Methods 2005, 148:78-87.

17. Deacon RM: Assessing nest building in mice. Nat Protoc 2006, 1:1117-1119.

18. Tillerson JL, Caudle WM, Reveron ME, Miller GW: Detection of behavioral impairments correlated to neurochemical deficits in mice treated with moderate doses of 1-methyl-4-phenyl-1,2,3,6-tetrahydropyridine. Exp Neurol 2002, 178:80-90.

19. Sedelis M, Schwarting RK, Huston JP: Behavioral phenotyping of the MPTP mouse model of Parkinson's disease. Behav Brain Res 2001, 125:109-125.

20. Rozas G, Lopez-Martin E, Guerra MJ, Labandeira-Garcia JL: The overall rod performance test in the MPTP-treated-mouse model of Parkinsonism. J Neurosci Methods 1998, 83:165-175.
21. Lee MS, Kim HS, Lyoo CH: "Off" gait freezing and temporal discrimination threshold in patients with Parkinson disease. Neurology 2005, 64:670-674.

22. Morris ME, Huxham F, McGinley J, Dodd K, lansek R: The biomechanics and motor control of gait in Parkinson disease. Clin Biomech (Bristol, Avon) 2001, 16:459-470.

23. Goldberg NR, Hampton T, McCue S, Kale A, Meshul CK: Profiling changes in gait dynamics resulting from progressive 1-methyl-4-phenyl-1,2,3,6tetrahydropyridine-induced nigrostriatal lesioning. J Neurosci Res 2011, 89:1698-1706.

24. Chastan N, Westby GW, Yelnik J, et al: Effects of nigral stimulation on locomotion and postural stability in patients with Parkinson's disease. Brain 2009, 132:172-184

25. Fernagut PO, Diguet $\mathrm{E}$, Labattu B, Tison F: A simple method to measure stride length as an index of nigrostriatal dysfunction in mice. J Neurosci Methods 2002, 113:123-130.

26. Klein A, Wessolleck J, Papazoglou A, Metz GA, Nikkhah G: Walking pattern analysis after unilateral 6-OHDA lesion and transplantation of foetal dopaminergic progenitor cells in rats. Behav Brain Res 2009, 199:317-325.

27. Amende I, Kale A, McCue S, et al: Gait dynamics in mouse models of Parkinson's disease and Huntington's disease. J Neuroeng Rehabil 2005, 2:20.

28. Vlamings R, Visser-Vandewalle V, Koopmans $G$, et al: High frequency stimulation of the subthalamic nucleus improves speed of locomotion but impairs forelimb movement in Parkinsonian rats. Neuroscience 2007, 148:815-823.

29. Madete JK, Klein A, Dunnett SB, Holt CA: Three-dimensional motion analysis of postural adjustments during over-ground locomotion in a rat model of Parkinson's disease. Behav Brain Res 2011, 220:119-125.

30. Schaafsma JD, Giladi N, Balash Y, et al: Gait dynamics in Parkinson's disease: relationship to Parkinsonian features, falls and response to levodopa. J Neurol Sci 2003, 212:47-53.

31. Vandeputte C, Taymans JM, Casteels C, et al: Automated quantitative gait analysis in animal models of movement disorders. BMC Neurosci 2010 11:92.

32. Tsung-Hsun H, Jia-JinJ C, Li-Hsien C, et al: Time-course gait analysis of hemiparkinsonian rats following 6-hydroxydopamine lesion. Behav Brain Res 2011, 222:1-9.

33. Chuang CS, Su HL, Cheng FC, et al: Quantitative evaluation of motor function before and after engraftment of dopaminergic neurons in a rat model of Parkinson's disease. J Biomed Sci 2010, 17:9.

34. Westin JE, Janssen MLF, Sager TN, Temel Y: Automated gait analysis in bilateral Parkinsonian rats and the role of L-DOPA therapy. Behav Brain Res 2012, 226:519-528

35. Moller KA, Berge OG, Hamers FPT: Using the CatWalk method to assess weight-bearing and pain behaviour in walking rats with ankle joint monoarthritis induced by carrageenan: Effects of morphine and rofecoxib. J Neurosci Methods 2008, 174:1-9.

36. Jackson-Lewis V, Przedborski S: Protocol for the MPTP mouse model of Parkinson's disease. Nat Protoc 2007, 2:141-151.

37. Kirik D, Rosenblad C, Bjorklund A: Characterization of behavioral and neurodegenerative changes following partial lesions of the nigrostriatal dopamine system induced by intrastriatal 6-hydroxydopamine in the rat. Exp Neurol 1998, 152:259-277.

38. Bergquist F, Shahabi HN, Nissbrandt H: Somatodendritic dopamine release in rat substantia nigra influences motor performance on the accelerating rod. Brain Res 2003, 973:81-91.

39. Tillerson JL, Miller GW: Grid performance test to measure behavioral impairment in the MPTP-treated-mouse model of parkinsonism. J Neurosci Methods 2003, 123:189-200.

40. Ogawa $N$, Hirose $Y$, Ohara $S$, Ono $T$, Watanabe $Y$ : A simple quantitative bradykinesia test in MPTP-treated mice. Res Commun Chem Pathol Pharmacol 1985, 50:435-441.

41. Hutter-Saunders JA, Kosloski LM, McMillan JM, et al: BL-1023 improves behavior and neuronal survival in 1-methyl-4-phenyl-1,2,3,6tetrahydropyridine-intoxicated mice. Neuroscience 2011, 180:293-304.

42. Crocker $\mathrm{SJ}$, Liston $\mathrm{P}$, Anisman $\mathrm{H}$, et al: Attenuation of MPTP-induced neurotoxicity and behavioural impairment in NSE-XIAP transgenic mice. Neurobiol Dis 2003, 12:150-161.

43. Sedelis M, Hofele K, Auburger GW, et al: MPTP susceptibility in the mouse: behavioral, neurochemical, and histological analysis of gender and strain differences. Behav Genet 2000, 30:171-182. 
44. Schwarting RK, Sedelis M, Hofele K, Auburger GW, Huston JP: Straindependent recovery of open-field behavior and striatal dopamine deficiency in the mouse MPTP model of Parkinson's disease. Neurotox Res 1999, 1:41-56.

45. Barbiero JK, Santiago RM, Lima MM, et al: Acute but not chronic administration of pioglitazone promoted behavioral and neurochemical protective effects in the MPTP model of Parkinson's disease. Behav Brain Res 2011, 216:186-192.

46. Schwarting RK, Huston JP: The unilateral 6-hydroxydopamine lesion model in behavioral brain research. Analysis of functional deficits, recovery and treatments. Prog Neurobiol 1996, 50:275-331.

47. Yurek DM, Deutch AY, Roth RH, Sladek JR Jr: Morphological, neurochemical, and behavioral characterizations associated with the combined treatment of diethyldithiocarbamate and 1-methyl-4-phenyl1,2,3,6-tetrahydropyridine in mice. Brain Res 1989, 497:250-259.

48. Chia LG, Ni DR, Cheng LJ, et al: Effects of 1-methyl-4-phenyl-1,2,3,6tetrahydropyridine and 5,7-dihydroxytryptamine on the locomotor activity and striatal amines in C57BL/6 mice. Neurosci Lett 1996, 218:67-71.

49. Bradbury AJ, Costall B, Jenner PG, et al: The effect of 1-methyl-4-phenyl1,2,3,6-tetrahydropyridine (MPTP) on striatal and limbic catecholamine neurones in white and black mice. Antagonism by monoamine oxidase inhibitors. Neuropharmacology 1986, 25:897-904.

50. Luchtman DW, Shao D, Song C: Behavior, neurotransmitters and inflammation in three regimens of the MPTP mouse model of Parkinson's disease. Physiol Behav 2009, 98:130-138.

51. German DC, Nelson EL, Liang CL, et al: The neurotoxin MPTP causes degeneration of specific nucleus $\mathrm{A} 8, \mathrm{~A} 9$ and $\mathrm{A} 10$ dopaminergic neurons in the mouse. Neurodegeneration 1996, 5:299-312.

52. Ricaurte GA, Langston JW, Delanney $L E$, et al: Fate of nigrostriatal neurons in young mature mice given 1-methyl-4-phenyl-1,2,3,6tetrahydropyridine: a neurochemical and morphological reassessment. Brain Res 1986, 376:117-124.

53. Ookubo M, Yokoyama $\mathrm{H}$, Kato $\mathrm{H}$, Araki T: Gender differences on MPTP (1-methyl-4-phenyl-1,2,3,6-tetrahydropyridine) neurotoxicity in C57BL/6 mice. Mol Cell Endocrinol 2009, 311:62-68.

54. Bezard E, Dovero S, Imbert C, Boraud T, Gross CE: Spontaneous long-term compensatory dopaminergic sprouting in MPTP-treated mice. Synapse 2000, 38:363-368.

55. Song DD, Haber SN: Striatal responses to partial dopaminergic lesion: Evidence for compensatory sprouting. J Neurosci 2000, 20:5102-5114

56. Kim ST, Son HJ, Choi JH, Ji IJ, Hwang O: Vertical grid test and modified horizontal grid test are sensitive methods for evaluating motor dysfunctions in the MPTP mouse model of Parkinson's disease. Brain Res 2010, 1306:176-183.

57. Blume SR, Cass DK, Tseng KY: Stepping test in mice: a reliable approach in determining forelimb akinesia in MPTP-induced Parkinsonism. Exp Neurol 2009, 219:208-211.

58. Neumann $M$, Wang $Y$, Kim S, et al: Assessing gait impairment following experimental traumatic brain injuryin mice. J Neurosci Methods 2009, 176:34-44.

59. Hoehn MM, Yahr MD: Parkinsonism: onset, progression and mortality. Neurology 1967, 17:427-442.

60. Morris M, lansek R, McGinley J, Matyas T, Huxham F: Three-dimensional gait biomechanics in Parkinson's disease: evidence for a centrally mediated amplitude regulation disorder. Mov Disord 2005, 20:40-50.

61. Yang YR, Lee YY, Cheng SJ, Lin PY, Wang RY: Relationships between gait and dynamic balance in early Parkinson's disease. Gait Posture 2008, 27:611-615

62. Knutsson E: An analysis of Parkinsonian gait. Brain 1972, 95:475-486.

63. Chee R, Murphy A, Danoudis M, Georgiou-Karistianis N, lansek R: Gait freezing in Parkinson's disease and the stride length sequence effect interaction. Brain 2009, 132:2151-2160.

64. Baltadjieva R, Giladi N, Gruendlinger L, Peretz C, Hausdorff JM: Marked alterations in the gait timing and rhythmicity of patients with de novo Parkinson's disease. Eur J Neurosci 2006, 24:1815-1820.

65. Snijders AH, Leunissen I, Bakker M, et al: Gait-related cerebral alterations in patients with Parkinson's disease with freezing of gait. Brain 2011 134:59-72.

66. Stolze $H$, Klebe $S$, Poepping $M$, et al: Effects of bilateral subthalamic nucleus stimulation on parkinsonian gait. Neurology 2001, 57:144-146.
67. Faist M, Xie J, Kurz D, et al: Effect of bilateral subthalamic nucleus stimulation on gait in Parkinson's disease. Brain 2001, 124:1590-1600.

68. Ebersbach G, Sojer M, Valldeoriola F, et al: Comparative analysis of gait in Parkinson's disease, cerebellar ataxia and subcortical arteriosclerotic encephalopathy. Brain 1999, 122(Pt 7):1349-1355.

69. Dibble LE, Nicholson DE, Shultz B, et al: Sensory cueing effects on maxima speed gait initiation in persons with Parkinson's disease and healthy elders. Gait Posture 2004, 19:215-225.

70. Burleigh Jacobs A, Horak FB, Nutt JG, Obeso JA: Step initiation in Parkinson's disease: Influence of levodopa and external sensory triggers. Mov Disord 1997, 12:206-215.

71. Giladi N, McDermott MP, Fahn S, et al: Freezing of gait in PD: prospective assessment in the DATATOP cohort. Neurology 2001, 56:1712-1721.

72. Chuang CS, Su HL, Cheng FC, et al: Quantitative evaluation of motor function before and after engraftment of dopaminergic neurons in a rat model of Parkinson's disease. J Biomed Sci 2010, 17:9.

doi:10.1186/1471-2202-13-142

Cite this article as: Wang et al:: Quantitative assessment of gait and neurochemical correlation in a classical murine model of Parkinson's disease. BMC Neuroscience 2012 13:142.

\section{Submit your next manuscript to BioMed Central and take full advantage of:}

- Convenient online submission

- Thorough peer review

- No space constraints or color figure charges

- Immediate publication on acceptance

- Inclusion in PubMed, CAS, Scopus and Google Scholar

- Research which is freely available for redistribution 